\title{
Results from GROCSE I: A Real-time Search for Gamma Ray Burst Optical Counterparts
}

\author{
Brian Lee ${ }^{1}$, Carl Akerlof ${ }^{1}$, Elden Ables ${ }^{2}$, Richard M. Bionta ${ }^{2}$, \\ Linda $\mathrm{Ott}^{2}$, Hye-Sook Park ${ }^{2}$, Eric Parker ${ }^{2}$, Scott Barthelmy ${ }^{3}$, \\ Paul Butterworth ${ }^{3}$, Thomas Cline ${ }^{3}$, Neil Gehrels ${ }^{3}$, Gerald \\ Fishman ${ }^{4}$, Chryssa Kouveliotou ${ }^{4}$, Charles Meegan ${ }^{4}$, Donald \\ Ferguson $^{5}$ \\ ${ }^{1}$ University Of Michigan, Ann Arbor, MI 48109 \\ ${ }^{2}$ Lawrence Livermore National Laboratory, Livermore, CA 94550 \\ ${ }^{3}$ NASA/Goddard Space Flight Center, Greenbelt, MD 20771 \\ ${ }^{4}$ NASA/Marshall Space Flight Center, Huntsville, AL 35812 \\ ${ }^{5}$ Earth Watch Inc., Pleasanton, CA 94550
}

The GROCSE I experiment (Gamma-Ray Optical Counterpart Search Experiment) is a rapid slewing wide field of view optical telescope at Lawrence Livermore National Laboratory, which responds to triggers from the BATSE GRB data telemetry stream that have been processed and distributed by the BACODINE network. GROCSE I has been in continuous automated operation since January 1994. As of October 1995, sky images for 22 GRB triggers have been recorded, in some cases while the burst was still in progress. The preliminary analysis of eight of these events are presented here. No optical counterparts have yet been detected. Limits for optical emission are given.

\section{INTRODUCTION}

Since January 1994, the GROCSE (Gamma-Ray Optical Counterpart Search Experiment) $(1,2,3,4)$ collaboration has been operating a wide-field-ofview automatic camera sited at Lawrence Livermore National Lab to search for optical counterparts corresponding to gamma ray bursts. GROCSE receives burst coordinates via BACODINE (BAtse COordinate DIstribution NEtwork) (5), which filters the telemetry data stream from BATSE in real time and transmits burst coordinate estimates to a Livermore computer via the internet. The camera then rapidly slews to the proper sky position, and obtains images of the GRB error box as soon as 12 seconds after BATSE's first detection - comparing this to a median burst duration of approximately 20 seconds for T90 (full width at 10\% maximum) (6), it's clear that GROCSE is able to record images of the GRB error box while many bursts are still in progress. GROCSE I has operated automatically every night, weather and

(C) 1996 American Institute of Physics 
equipment permitting, since its activation in January 1994, to date recording twenty-two confirmed GRB events in over 3600 hours of operating time.

\section{THE GROCSE I INSTRUMENT}

In the summer of 1993 we initiated the GROCSE collaboration to adapt a camera originally designed for the SDI program to the task of detecting GRB's. The current star imaging system consists of a single 52.5 degree wide-field-of-view (WFOV) lens, 23 intensified CCD imaging devices, and associated readout electronics. The $\mathrm{f} / 2.8$ lens, manufactured by Perkin-Elmer, has an effective aperture of $89 \mathrm{~mm}$, and a focal length of $250 \mathrm{~mm}$. The components of the lens are solid blocks of glass with concentric spherical surfaces. The spherical focal plane is imaged through 23 custom machined reducing fiber optic bundles. Glued to the back end of each fiber optic reducer is an image intensifier, and the optical output of each intensifier is transmitted through a second reducing fiber optic bundle to a $384 \times 576$ CCD with $23 \mu \mathrm{m}$ pixels, covering a $7.5 \times 11.5$ degree field of view. The total image reduction is $3.8: 1$, with each pixel covering a 1.2 arc-min field of view. The 23 cameras cover $75 \%$ of the lens' field of view for an effective solid angle of 0.621 steradians. The exposure duration is set by thumbwheel switches to 0.5 seconds. The entire assembly is mounted on a Contraves computer-controlled inertial guidance test system which has maximum angular slew rates of $100^{\circ} / \mathrm{sec}$ and $200^{\circ} / \mathrm{sec}$ for the azimuth and elevation axes. The analog CCD output from each camera is connected to a Datacube image processing system, under the control of a Sun 4/330 host computer. The GROCSE data acquisition system runs entirely automatically each night, imaging the entire sky every 30 minutes and responding to any BACODINE triggers within its field of regard.

\section{DATA ANALYSIS}

Analysis of the GROCSE I data requires several steps. First our code must perform a "pseudo field flattening" to correct for variations of intensifier sensitivity across the image. (Since intensifier response varies with gain, and the gain is automatically adjusted by the online code for peak image quality, we can not use a true flat field.) The code then locates the positions of all "blobs" (starlike clusters of bright pixels $5 \sigma$ above the background) in the image. Using previously determined camera alignment parameters and functions which remove the 2-D distortions caused by the image intensifiers and reducer bundles, the complete coordinate transform code then automatically determines absolute RA and DEC coordinates of each blob in all of the images. The code can then identify objects by comparison to other images and to know stars from the SAO star catalog. Any unidentified, new, or unusual objects are tagged as candidates for further investigation. Under ideal conditions (a moonless, clear night) this system can reliably identify stars as dim as $M_{\mathrm{V}}=8.5$. 


\section{RESULTS}

As of October 1995 we have recorded sky images for 22 BATSE GRB triggers. BACODINE sends two kinds of burst triggers; "type 1" triggers, which we receive within approximately 6 seconds of the BATSE detection, and "type 11 " triggers which arrive some 10 minutes later. Approximately half of these 22 triggers are useful for later analysis, as clouds and the large BACODINE error box have often interfered with our observations. Prior to 1995 GROCSE I used only the 7 central cameras in burst mode. It now uses all 23 cameras, greatly increasing our coverage of many events but also increasing the time between consecutive images.

We have analyzed eight of the type 1 triggers, scanning the camera or cameras containing the $1 \sigma$ BATSE error box for transients (Figure 1.). Three of these observations were simultaneous with the BATSE $\gamma$-ray detection, the other five were within seconds after the BATSE signal ended. The image closest in time to the burst (and in three cases simultaneous with the burst) was compared first to the $\mathrm{SAO}$ catalog, effectively masking out a $5 \times 5$ pixel box around any SAO star. Unidentified or new objects were then compared to all later burst mode images, sky patrol images, and other catalogs. All nonSAO objects were studied and eventually identified as bad pixels, satellites, or known celestial objects, eliminating any possible candidates for optical counterparts.

The failure to detect an optical transient provides us with an upper limit on any possible optical emission at the time of the image, based on the limiting visual magnitude of stars in the image. This limiting $M_{\mathrm{V}}$ for each observation was calculated by comparison of observed stars to the SAO catalog. At our quoted limit an object of that brightness or brighter would be detected with approximately a $90 \%$ probability. In most cases, some stars one or more visual magnitudes dimmer that the quoted limit are visible in the image.

We report this result in terms of the $\gamma$-ray to optical flux ratio. We have calculated this ratio in two ways: for all observations, we report a total $\gamma$-ray fluence to one second optical fluence limit, using the historical assumption of a one second optical flash. This number makes no assumptions as to when a flash will occur. For the three simultaneous observations, we also report an instantaneous $\gamma$-ray flux to optical flux ratio, which places a limit on any optical signal occurring in coincidence with the BATSE signal. Results are in Table 1.

\section{CONCLUSIONS}

To date GROCSE I has not detected any counterparts, with limiting $M_{\mathrm{V}}$ 's up to 8.5. This, combined with the number of GROCSE I observations, places convincing limits on the $\gamma$-ray to optical light ratio of a GRB. This result is consistent with the predictions of some theories - Band and Ford (8) extrapolate the GRB spectra to the optical, and predict that only the very brightest GRBs might be visible by GROCSE I, with a median burst 


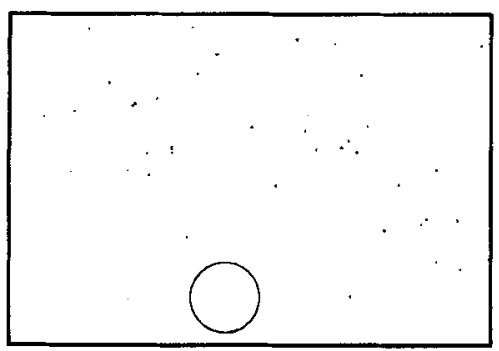

GRB 3040 06/23/94

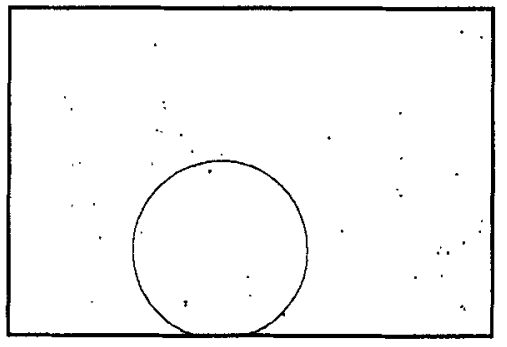

GRB $3141 \quad 08 / 28 / 94$

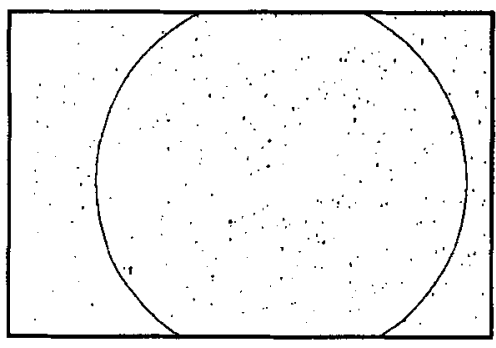

GRB 3159 09/07/94

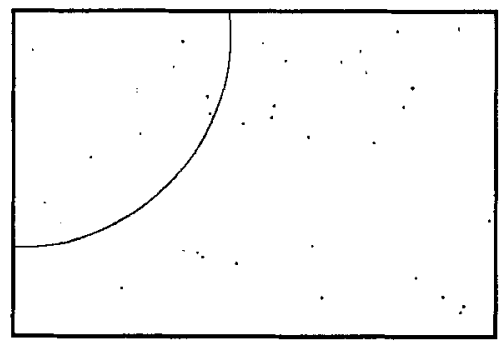

GRB $361105 / 31 / 95$ (One of five cameras)

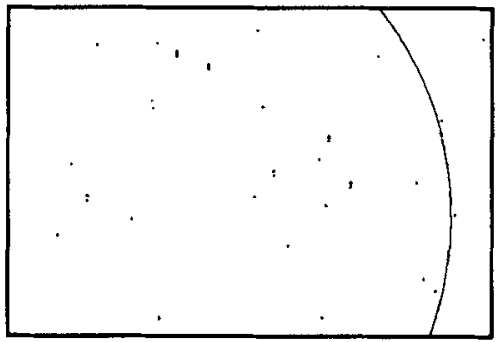

GRB $377909 / 07 / 95$ (One of three cameras)

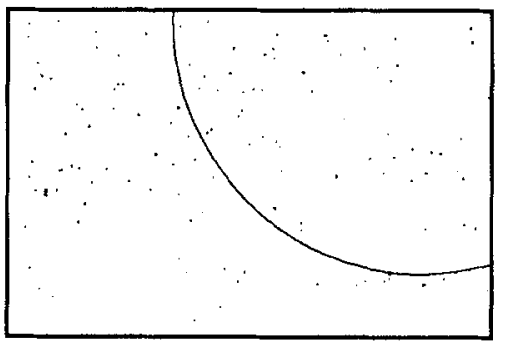

GRB $380509 / 18 / 95$

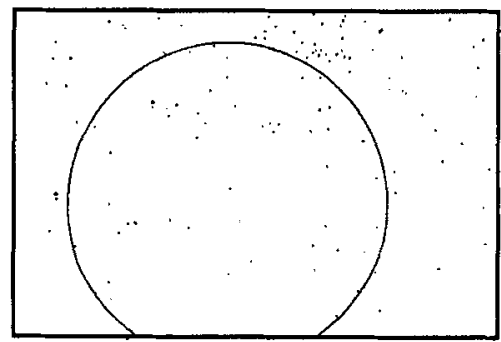

GRB 3814 09/22/95 (One of two cameras)

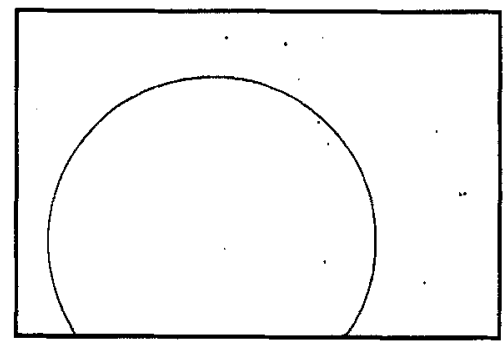

FIG. 1. GROCSE I coverage of the $1 \sigma$ BATSE error box.

having a visible magnitude closer to 14 . The GROCSE collaboration is now completing work on a second instrument, GROCSE II (7) which is able to detect magnitude 14 objects, corresponding to a $\gamma$-ray to optical energy ratio of $10^{6}$ or more for brighter bursts. 
TABLE 1. GROCSE I Results

\begin{tabular}{|c|c|c|c|c|c|c|c|c|c|c|}
\hline $\begin{array}{l}\text { BATSE } \\
\text { Trigger }\end{array}$ & UTC Datc & \begin{tabular}{|l} 
First \\
Image \\
(sec)
\end{tabular} & $\begin{array}{l}\text { BATSE } \\
\mathrm{T}_{\boldsymbol{n}} \\
\text { (sec) }\end{array}$ & \begin{tabular}{|c|}
$M_{\mathrm{v}}$ \\
Limit
\end{tabular} & $\begin{array}{c}\text { Optical flux } \\
\text { Limit } \\
(\text { ergs/cm²sec) } \\
\cdot 1^{-9}\end{array}$ & $\begin{array}{c}\text { Percent } \\
\text { Coverage } \\
\text { (lo error } \\
\text { box) }\end{array}$ & $\begin{array}{c}50.300 \mathrm{keV} \\
\text { Total } \mathrm{F}_{\mathcal{Y}} \\
\left(\mathrm{ergs} / \mathrm{cm}^{2}\right) \\
\cdot 10^{-7}\end{array}$ & $\begin{array}{l}\text { Total F } \\
\text { i/ sec } \\
\text { F pelced } \\
\text { Limit }\end{array}$ & $\begin{array}{c}\text { Instantaneous } \\
y-F l u x \\
\left(\mathrm{ergs} / \mathrm{cm}^{2} \mathrm{sec}\right) \\
\cdot 10^{-8}\end{array}$ & $\begin{array}{c}\text { Instantaneous } \\
\text { y-Flux ! } \\
\text { Optical Flux } \\
\text { Limit }\end{array}$ \\
\hline \multicolumn{11}{|c|}{ Simultaneous Observations } \\
\hline 3040 & $06 / 23 / 94$ & 24 & 26 & 7.3 & 12.0 & $98 \%$ & 20.5 & 170 & 7.3 & 6.1 \\
\hline 3159 & $09 / 07 / 94$ & 22 & 18 & 7.0 & 15.8 & $30 \%$ & 6.20 & 39 & $5 . \overline{3}$ & 3.3 \\
\hline 3805 & $09 / 18 / 95$ & 20 & 40 & 7.7 & 8.25 & $95 \%$ & 6.62 & 80 & 3.1 & 3.8 \\
\hline \multicolumn{11}{|c|}{ Rapid Followup Obscrvations } \\
\hline 2793 & $01 / 29 / 94$ & 35 & 7 & 7.3 & 12.0 & $99 \%$ & 3.40 & 280 & & \\
\hline 3141 & $08 / 28 / 94$ & 21 & 2 & 8.5 & 3.95 & $94 \%$ & 1.77 & 45 & & \\
\hline 3611 & $05 / 31 / 95$ & 23 & 3 & 7.1 & 14.3 & $-75 \%$ & 1.07 & 7.5 & & \\
\hline 3779 & $09 / 07 / 95$ & 35 & 7 & 7.5 & 9.90 & $-75 \%$ & 1.94 & 20 & & \\
\hline 3814 & $09 / 22 / 95$ & 46 & 5 & 7.0 & 15.8 & $90 \%$ & 28.4 & 180 & & \\
\hline
\end{tabular}

\section{REFERENCES}

1. H-S. Park, et al., SPIE Conf. Proc. 1111, 196 (1989).

2. H-S. Park, et al., SPIE Conf. Proc. 1304, 293 (1990).

3. C. Akerlof, et al., in Gamma-Ray Bursts, eds. G.J.Fishman, J.J. Brainerd \& K. Hurley, AIP Conf. Proc. 307, 633 (AIP, New York, 1994).

4. C. Akeriof, et al., Astrophys. Sp. Sci. 231(1-2), 255 (1995).

5. S. Barthelmy, et al., in Gamma-Ray Bursts, eds. G.J.Fishman, J.J. Brainerd \& K. Hurley, AIP Conf. Proc. 307, 643 (AIP, New York, 1994).

6. C. Kouveliotou, et al., in Gamma-Ray Bursts, eds. G.J.Fishman, J.J. Brainerd \& K. Hurley, AIP Conf. Proc. 307, 167 (AIP, New York, 1994).

7. H-S. Park, et al., these proceedings (1996).

8. D.Band \& L. Ford, Astrophys. Sp. Sci. 231(1-2), 247 (1995). 